\title{
Population dynamics and rapid spread of Cardinium, a bacterial endosymbiont causing cytoplasmic incompatibility in Encarsia pergandiella (Hymenoptera: Aphelinidae)
}

\author{
LR Harris ${ }^{1}$, SE Kelly ${ }^{2}$, MS Hunter ${ }^{2}$ and SJ Perlman ${ }^{1}$ \\ ${ }^{1}$ Department of Biology, University of Victoria, Victoria, British Columbia, Canada and ${ }^{2}$ Department of Entomology, The University \\ of Arizona, Tucson, AZ, USA
}

\begin{abstract}
Cytoplasmic incompatibility $(\mathrm{Cl})$ is a common phenotype of maternally inherited bacterial symbionts of arthropods; in its simplest expression, uninfected females produce few or no viable progeny when mated to infected males. Infected females thus experience a reproductive advantage relative to that of uninfected females, with the potential for the symbiont to spread rapidly. $\mathrm{Cl}$ population dynamics are predicted to depend primarily on the strength of incompatibility, the fitness cost of the infection and how faithfully symbionts are inherited. Although the bacterial symbiont lineage Wolbachia has been most identified with the $\mathrm{Cl}$ phenotype, an unrelated bacterium, Cardinium may also cause $\mathrm{Cl}$. In the first
\end{abstract}

examination of population dynamics of $\mathrm{Cl}$-inducing Cardinium, we used population cages of the parasitic wasp Encarsia pergandiella (Hymenoptera: Aphelinidae) with varying initial infection frequencies to test a model of invasion. Cardinium was found to spread rapidly in all populations, even in cases where the initial infection frequency was well below the predicted invasion threshold frequency. The discrepancy between the modeled and actual results is best explained by weaker $\mathrm{Cl}$ than measured in the lab and a cryptic fitness benefit to the infection.

Heredity (2010) 104, 239-246; doi:10.1038/hdy.2009.130; published online 7 October 2009

Keywords: reproductive manipulation; symbiosis; endosymbiont; fitness benefit; Wolbachia; population cage

\section{Introduction}

A large number of insect species harbor maternally inherited bacterial symbionts that have diverse effects on their hosts (Moran et al., 2008). Many symbionts are beneficial to their hosts, providing them with essential nutrients (Buchner, 1965; Baumann, 2005) or protection from parasitoids or pathogens (Oliver et al., 2003; Scarborough et al., 2005). On the other hand, some symbionts are known as reproductive manipulators, and do not confer direct benefits to their hosts, but instead manipulate their host's reproduction in ways that increase their transmission.

The most common reproductive manipulation strategy is cytoplasmic incompatibility (CI), whereby uninfected females produce few or no viable progeny when mated with infected males. Infected females, in contrast, can mate with either uninfected or infected males without negative effects. In this way, infected females experience a reproductive advantage relative to that of uninfected females, and rapid spread of the CI symbiont can result (Caspari and Watson, 1959; Turelli and Hoffmann, 1991; Engelstadter and Telschow, 2009). The ability of CI

Correspondence: LR Harris, Department of Biology, University of Victoria, 3800 Finnerty Rd, Petch Building 116, Victoria, British Columbia V8P 5C2, Canada.

E-mail: leanner.harris@gmail.com

Received 8 June 2009; revised 31 August 2009; accepted 1 September 2009; published online 7 October 2009 symbionts to spread has many potential applications, including population suppression or the introduction of beneficial genes into an insect population (Sinkins et al., 1997; Zabalou et al., 2004; Xi et al., 2005; Brelsfoard et al., 2008; McMeniman et al., 2009).

Two bacterial endosymbionts have been found to be able to induce CI in arthropods. Wolbachia, in the $\alpha$-proteobacteria, is the best known reproductive manipulator, infecting at least $16-24 \%$ of all arthropod species (Werren et al., 1995; Weeks et al., 2003; Zchori-Fein and Perlman, 2004) and potentially as many as $66 \%$, when low prevalence infections are considered (Hilgenboecker et al., 2008). Wolbachia has been shown to cause CI in all of the major insect orders, as well as in mites and isopods (Stouthamer et al., 1999). More recently, Cardinium, a member of the phylum Bacteroidetes, has been found to cause CI in three hosts: in the parasitoid wasp Encarsia pergandiella (Hunter et al., 2003), and in the spider mites Eotetranychus suginamensis and Bryobia sarothamni (Gotoh et al., 2007; Ros and Breeuwer, 2009). Since its discovery, Cardinium has been found in four orders and approximately 6-7\% of arthropods (Weeks et al., 2003; Zchori-Fein and Perlman, 2004; Duron et al., 2008). Wolbachia and Cardinium are primarily vertically transmitted (Hoffmann et al., 1990; Perlman et al., 2008), although rare horizontal transmission events are thought to occur on an evolutionary timescale (Werren, 1997; Zchori-Fein and Perlman, 2004). 
The spread and equilibrium frequency of a CIinducing symbiont within a population will depend on its relative costs and benefits to the host (Caspari and Watson, 1959; Turelli, 1994; Turelli and Hoffmann, 1995; Vavre et al., 2000). The symbiont's ability to induce incompatible matings will facilitate its spread within a population, because infected females experience a reproductive advantage relative to uninfected females. However, if there is a cost associated with the infection, for example, decreased fecundity, this will reduce the symbiont's ability to spread. Consequently, the infection is expected to spread when the benefits to hosts of being infected (that is, higher reproductive success) outweigh the fitness costs. Although fitness costs, such as reduced fecundity, are not expected to vary with infection frequency, the reproductive benefits associated with the infection are frequency dependent. In a population with a high infection frequency, a female is very likely to mate with an infected male, and thus benefits greatly from being infected. However, in a population where the infection is rare, a female is not very likely to mate with an infected male, and therefore receives little benefit in harboring the infection. At low frequency, therefore, fecundity costs might be expected to outweigh potential reproductive benefits.

CI invasion and spread are thought to depend primarily on three key parameters: any losses in maternal transmission $(\mu)$, the relative fitness of infected females compared to uninfected females $(F)$ and the offspring production of incompatible crosses relative to compatible crosses $(H)$ (Caspari and Watson, 1959; Turelli, 1994; Turelli and Hoffmann, 1995; Vavre et al., 2000). Models of CI dynamics make two key predictions. First, the equilibrium frequency of infection is predicted to be stable in a population at two points: when the infection is at or near fixation and when it is completely lost from the population (Turelli, 1994). Second, an unstable equilibrium, also termed the invasion threshold, is found between these two stable infection frequencies. When the frequency of infection is higher than the invasion threshold, the infection is expected to spread until it is at or near fixation. Alternatively, if the infection frequency is lower than the invasion threshold, the symbiont will be lost from the population (Turelli, 1994).

Understanding the invasion and spread of CI symbionts is critical for the successful application of $\mathrm{CI}$ for disease and pest control (Rasgon, 2008). Moreover, virtually no studies to date have tested theoretical models of CI spread, and such studies are essential to link our theoretical knowledge of CI with empirical data (Engelstadter and Telschow, 2009). In addition, cytoplasmic incompatibility models may be inaccurate if other factors are influencing CI spread, such as population subdivision or cryptic fitness costs or benefits (Egas et al., 2002). For instance, $\mathrm{Xi}$ et al. (2005) used population cages to monitor the spread of CI Wolbachia within a mosquito population. They found that Wolbachia did not spread until the initial infection frequency was well above the predicted invasion threshold, suggesting that there was a cryptic fitness cost associated with the infection that was not revealed in laboratory assays of individuals (Xi et al., 2005).

In the parasitoid wasp E. pergandiella, the CI symbiont Cardinium was found to induce a high fitness cost, resulting in a relatively high predicted invasion threshold of 20-24\% (Perlman et al., 2008). The prediction of a high invasion threshold is surprising, because field populations are found at or near fixation (Perlman et al., 2008). To examine this prediction, we established E. pergandiella populations with varying initial Cardinium infection frequencies and monitored the dynamics of the infection for nine generations. In addition to testing a model of CI invasion using laboratory estimates of parameter values, this study allowed us to more accurately estimate the threshold frequency and host fitness effects of Cardinium under more realistic conditions.

\section{Methods}

\section{Cultures}

E. pergandiella is a solitary hymenopteran parasitoid of whiteflies with an unusual 'autoparasitic' biology (Hunter and Woolley, 2001); females lay single female eggs in whitefly nymphs, whereas male eggs are laid in developing wasp larvae or pupae, either their own species, or other primary parasitoids, enclosed within the whitefly cuticle. The infected line was collected from Bemisia tabaci hosts in the Rio Grande Valley in Texas in 2003, and was maintained in the laboratory on B. tabaci reared on cowpea plants (Vigna unguiculata). This line is fixed for Cardinium infection and is not infected with Wolbachia (Hunter et al., 2003). The infection is primarily maternally transmitted, and a previous study by Perlman et al. (2008) suggests that paternal and/or horizontal transmission is absent or rare. An uninfected line was obtained by curing a subpopulation of the infected line by treating adult wasps with $50 \mathrm{mg} \mathrm{ml}^{-1}$ rifampicin in honey for three generations.

\section{Strength of cytoplasmic incompatibility}

The number of offspring produced by the incompatible cross relative to the compatible cross will strongly influence the invasion and spread of a CI symbiont (Turelli, 1994). To determine the strength of CI in Cardinium-infected E. pergandiella, we mated freshly emerged uninfected virgin females individually to 1- to 2-day-old virgin males of known infection status. Each female was then transferred to a $35 \mathrm{~mm}$ Petri dish containing $1 \%$ agar, upon which a cowpea leaf disk was placed. Females were allowed to oviposit on leaf disks infested with 50-100 whiteflies in the third to early fourth nymphal stage for $24 \mathrm{~h}$.

Following the oviposition period, infested leaves were incubated at $27^{\circ} \mathrm{C}$ until E. pergandiella pupae could be counted and removed (8-10 days). When an E. pergandiella egg fails to develop due to $\mathrm{CI}$, the parasitized whitefly is developmentally arrested (Hunter et al., 2003). Therefore, the number of arrested whiteflies served as an indicator of CI. Whiteflies were considered developmentally arrested if, by the time of scoring, they had not developed eyespots or wing buds. The majority of whiteflies in the control treatment had already emerged at the time of scoring. Females that did not produce any pupae and produced $<2$ whiteflies scored as developmentally arrested were presumed to be unmated and were removed from the analysis.

The average reduction in the number of pupae produced by the incompatible cross (infected 
male $\times$ cured female) relative to the average number of pupae produced by the compatible cross (cured male $\times$ cured female) was measured as $H$.

\section{Invasion models}

The spread of Cardinium was modeled using the Hoffmann-Turelli model (Turelli, 1994; Turelli and Hoffmann, 1995), modified by Vavre et al. (2000) for haplodiploid hosts with the female mortality type of CI:

$$
\begin{aligned}
f_{t+1} & =\frac{F f_{t}(1-\mu)}{F f_{t}\left[1-\mu(1-H) m_{t}\right]+\left(1-f_{t}\right)\left[1-(1-H) m_{t}\right]} \\
m_{t+1} & =\frac{F f_{t}(1-\mu)}{F f_{t}+\left(1-f_{t}\right)}
\end{aligned}
$$

where $f_{t}$ is the female infection frequency at time $t, f_{t+1}$ the female infection frequency one generation after time $t, F$ the number of offspring produced by infected females relative to uninfected females and can thus describe a fitness cost $(F<1)$ or a fitness benefit $(F>1)$ of the infection, $\mu$ the loss in maternal transmission efficiency, $H$ the proportion of offspring produced by the incompatible cross relative to the compatible cross, $m_{t}$ the male infection frequency at time $t$ and $m_{t+1}$ the male infection frequency one generation after time $t$. The invasion threshold frequency was calculated using equation A5 in Vavre et al. (2000).

\section{Population cages}

E. pergandiella wasps of known infection status were introduced to $50 \mathrm{~cm}^{3}$ cages (four cages per treatment) to establish populations with low (15\%), medium (36\% for females and $31 \%$ for males) and high (55\%) initial infection frequencies. The infection frequencies used to establish the medium treatment were at the predicted invasion threshold, when using the following laboratory estimates of infection parameter values. In a previous study, Cardinium-infected female E. pergandiella produced $18 \%$ fewer offspring than uninfected females when supplied with unlimited whitefly hosts over a period of 4 days (Perlman et al., 2008). Therefore, as an upper limit to $F$, we estimated the fitness cost of the infection to be $18 \%(F=0.82)$. Maternal transmission efficiency was nearly perfect among field-caught females (Perlman et al., 2008), so we used the conservative estimate of 99\% $(\mu=0.01)$. In this study we found that, on average, the incompatible cross produced $38 \%$ of the offspring of the control cross $(H=0.38)$. Given these parameter estimates, we predicted that the invasion frequency required for Cardinium to spread was $36 \%$ for females and $31 \%$ for males.

The initial infection frequencies were established using three successive weekly introductions of 50 female and 24-33 male adult wasps. All wasps were 3-5 days old at the time of introduction. Because E. pergandiella is autoparasitic, plants bearing whitefly hosts for female eggs and heterospecific parasitoid pupae for male eggs were both provided in the cages. The majority of whiteflies were third instar nymphs at the time that plants were introduced to the cages, whereas the majority of the parasitoids, Eretmocerus eremicus, were early pupae. Each week, wasps were provided with two cowpea plants infested with $B$. tabaci and an additional plant infested with E. eremicus developing on B. tabaci. Plants were removed from the cages before adult wasps of either E. pergandiella or E. eremicus emerged, the leaves placed in an emergence jar and a subset of 50 female and 30 adult males from the new generation were reintroduced to the cage. In generation three, there was a shortage of males in some cages due to the poor health of plants infested with E. eremicus. Therefore, male introductions in two cages of the 'low treatment', two cages of the 'medium treatment' and one cage of the 'high treatment' were supplemented with 30, 30, 25, 13 and 8 males from the previous week of the same cage, respectively. We expect the effect of using males of the previous week to be minimal and, if anything, to slow down any increases in infection. Infection frequencies in each cage were estimated using PCR at generations 2, 4, 6,8 and 9 (see below). Wasps were frozen or stored in 95\% ethanol until DNA extraction.

\section{DNA extractions}

To assess Cardinium infection, we performed single wasp DNA extractions on at least 50 female E. pergandiella wasps from each cage for each generation tested. DNA was extracted by grinding individual wasps in $3 \mu \mathrm{l}$ of $20 \mathrm{mg} \mathrm{ml}^{-1}$ proteinase $\mathrm{k}$, and adding the homogenate to $50 \mu \mathrm{l}$ of $5-10 \% \mathrm{w} / \mathrm{v}$ Chelex (White et al., 2009). Samples were incubated at $37^{\circ} \mathrm{C}$ for $1 \mathrm{~h}$ and then at $96^{\circ} \mathrm{C}$ for $8 \mathrm{~min}$, with periodic vortexing. Samples were stored at $-20{ }^{\circ} \mathrm{C}$ for a maximum of 3 months.

\section{Diagnostic PCR}

Wasps were screened for the presence of Cardinium using diagnostic PCR. Two sets of Cardinium-specific primers were used, Ch441F (GTACAGGAGCAAAACAATCCC) and either Ch665R (TATTCTTAACTCAAGCCTAAT) or Ch1017R (ATTTTTCAAAGTAGCAAAATA), which amplified an $\sim 200$ - and 600-bp region of 165 rDNA, respectively (developed by Stephan Schmitz-Esser, University of Vienna). PCR conditions for $\mathrm{Ch} 441 \mathrm{~F}$ and Ch665R were a $3 \mathrm{~min}$ initial denaturation at $94{ }^{\circ} \mathrm{C}$, followed by 40 cycles of $94{ }^{\circ} \mathrm{C}$ for $30 \mathrm{~s}, 53^{\circ} \mathrm{C}$ for $45 \mathrm{~s}$ and $72{ }^{\circ} \mathrm{C}$ for $45 \mathrm{~s}$, and a final extension of $72{ }^{\circ} \mathrm{C}$ for $5 \mathrm{~min}$. PCR conditions for $\mathrm{Ch} 441 \mathrm{~F}$ and Ch1017R were a $3 \mathrm{~min}$ initial denaturation at $94{ }^{\circ} \mathrm{C}$, followed by 40 cycles of $94{ }^{\circ} \mathrm{C}$ for $30 \mathrm{~s}, 56{ }^{\circ} \mathrm{C}$ for $45 \mathrm{~s}$ and $72{ }^{\circ} \mathrm{C}$ for $1 \mathrm{~min}$, and a final extension of $72{ }^{\circ} \mathrm{C}$ for $6 \mathrm{~min}$. Samples that appeared to be negative for Cardinium were screened twice more. A positive control of an infected E. pergandiella and a negative control of a cured $E$. pergandiella were included in each run.

Samples determined to be negative for Cardinium were screened for single- or double-copy E. pergandiella genes (EF1 $\alpha$, opsin or histone) as a positive control for the DNA extraction. An 380-bp segment of EF1 $\alpha$ was amplified using EpergEF-F and EpergEF-R (Perlman et al., 2008) with PCR conditions of $95^{\circ} \mathrm{C}$ for $3 \mathrm{~min}$, followed by 30 cycles of $95^{\circ} \mathrm{C}$ for $60 \mathrm{~s}, 50{ }^{\circ} \mathrm{C}$ for $60 \mathrm{~s}$ and $72{ }^{\circ} \mathrm{C}$ for $90 \mathrm{~s}$, with a final extension of $72{ }^{\circ} \mathrm{C}$ for $10 \mathrm{~min}$. An $\sim 1.5-\mathrm{kb}$ fragment of the opsin gene was amplified using LWRh-F and LWRh-R according to Mardulyn and Cameron (1999), and a faint band of $\sim 700 \mathrm{bp}$ was often also observed, likely because this gene is duplicated in Hymenoptera. An $\sim 400-b p$ region of histone was amplified using H3Af and H3Ar (Colgan et al., 1998) 
with PCR conditions of $94^{\circ} \mathrm{C}$ for $3 \mathrm{~min}$, followed by 40 cycles of $94{ }^{\circ} \mathrm{C}$ for $45 \mathrm{~s}, 65^{\circ} \mathrm{C}$ for $45 \mathrm{~s}$ and $72{ }^{\circ} \mathrm{C}$ for $60 \mathrm{~s}$, with a final extension of $72{ }^{\circ} \mathrm{C}$ for $6 \mathrm{~min}$. A positive control of E. pergandiella and a negative no-DNA control were included in each EF1 $\alpha$, opsin and histone PCR. Samples that appeared negative for E. pergandiella DNA were excluded from further analysis. At least one PCR product of each gene was sequenced for confirmation (Macrogen Inc., Seoul, South Korea). For sequencing, all PCR products were purified using a QIAquick PCR purification kit (Qiagen), except for opsin, which was first cloned into Escherichia coli using a StrataClone PCR cloning kit (Stratagene, La Jolla, CA, USA). Novel opsin and histone sequences were deposited in GenBank under the accession numbers FJ483848 and FJ842099, respectively.

\section{Statistical analysis}

A Mann-Whitney test was used to determine if wasp offspring number was significantly different between the predicted incompatible and compatible crosses. To determine if there were significant changes in infection frequency over the nine-generation period, we calculated a replicated goodness-of-fit test. Logistic regression analysis was performed using the binomial response variable of the presence or absence of infection within individual wasps, where each population cage was nested within treatment (SAS Institute, Cary, NC, USA). This analysis allowed for the identification of variables that were significant predictors of the probability of infection with Cardinium. The Hosmer and Lemeshow goodness-of-fit test was used to examine fit between the logistic regression model and the data.

To determine the infection parameter values of the model that best fit our observed data, we simultaneously varied all three parameters $(0 \leqslant F \leqslant 2 ; 0 \leqslant H \leqslant 1 ; 0 \leqslant \mu \leqslant 1)$ using increments of 0.01 , and calculated the global sum of squares between the modeled predictions and the observed infection frequencies for all treatments. We selected the parameter values of best fit as the combination of values that resulted in the lowest sum of squares (MATLAB 7.4; The MathWorks, MA, USA). We also determined the parameter values that best fit the observed data when $H$ was fixed at the experimentally determined value of 0.38 in a similar fashion.

\section{Results}

Wasp offspring number was significantly lower when uninfected females were mated with infected males (that is, the incompatible cross) compared with when they were mated with uninfected males (that is, the compatible cross; Mann-Whitney test; $U=55.5 ; \quad n=47$; $P<0.0001)$. On average, incompatible crosses produced $38 \%$ of the offspring produced by the compatible crosses $(H=0.38)$.

Cardinium was found to spread rapidly in E. pergandiella for all three initial infection frequencies (Figure 1). Initial infection frequencies were significantly different from the infection frequencies at generation nine for the treatment below $\left(G_{\mathrm{p}}=111.37 ; \mathrm{df}=1 ; P<0.001\right)$, at $\left(G_{\mathrm{p}}\right.$ $=257.45 ; \mathrm{df}=1 ; \quad P<0.001)$ and above the predicted invasion threshold $\left(G_{p}=176.28 ; \mathrm{df}=1 ; P<0.001\right)$. There was considerable variation in infection frequencies between cages within treatments (Figure 1), and this

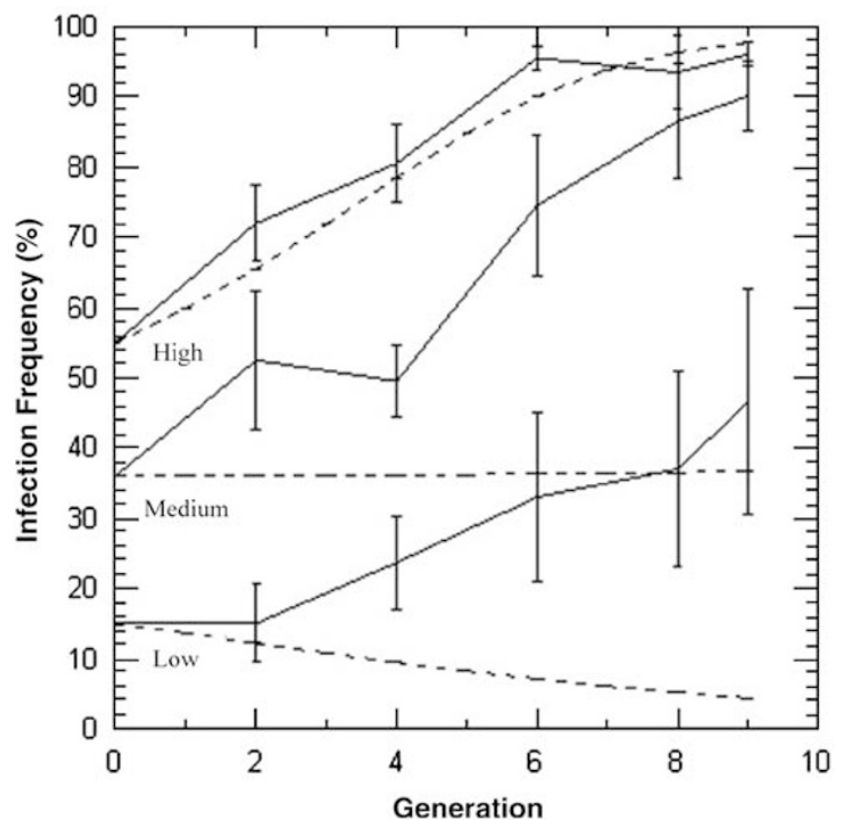

Figure 1 Spread of the bacterial symbiont Cardinium in Encarsia pergandiella population cages with initial infection frequencies of 15 , 36 and 55\% (solid lines) compared with predicted spread based on infection parameter estimates of fitness cost $F=0.82$, maternal transmission efficiency $\mu=0.01$ and cytoplasmic incompatibility (CI) strength $H=0.38$ (dotted lines). Data represent the mean \pm standard error, $n=4$.

was particularly true in generation 2, where the infection frequency of one cage in the medium treatment was $29 \%$ higher than the average for the other cages in this treatment. Generation $(P<0.001)$, treatment $(P<0.001)$, cage $(P<0.002)$ and the interaction between generation and cage $(P<0.0001)$ were significant predictors of the probability of Cardinium infection. The logistic regression model generated using these parameters showed a good fit to the data (Hosmer and Lemeshow goodness-of-fit test $\left.\chi^{2}=9.01 ; \mathrm{df}=8 ; P>0.34\right)$. The interaction between treatment and generation was not associated with the probability of infection (logistic regression; $P>0.05$ ).

The observed data best fit a model with a fitness benefit of $F=1.09$, a loss in maternal transmission of $\mu=0.01$ and a reduction in incompatible offspring of $H=0.61$ (Figures $2 \mathrm{a}$ and $3 \mathrm{a}$ ). When $H$ was fixed to the experimentally determined value of 0.38 , an optimal fit to the observed data was found using a fitness benefit $(F=1.12)$ and a loss in maternal transmission of $\mu=0.07$ (Figures $2 b$ and $3 b$ ). Although these parameter value combinations fit optimally, a range of parameter values provided good fit to the observed data (that is, sum of squares $<1$ ), as seen in Figure 3.

\section{Discussion}

Cardinium was found to spread rapidly in E. pergandiella for all initial infection frequencies (Figure 1), suggesting that the invasion threshold is much lower than that of our modeled predictions. Although this is the first study on population dynamics of Cardinium-induced CI, a few studies have documented the invasion and spread of $\mathrm{CI}$ Wolbachia. The rapid increase in infection prevalence observed for Cardinium in E. pergandiella is similar to that 

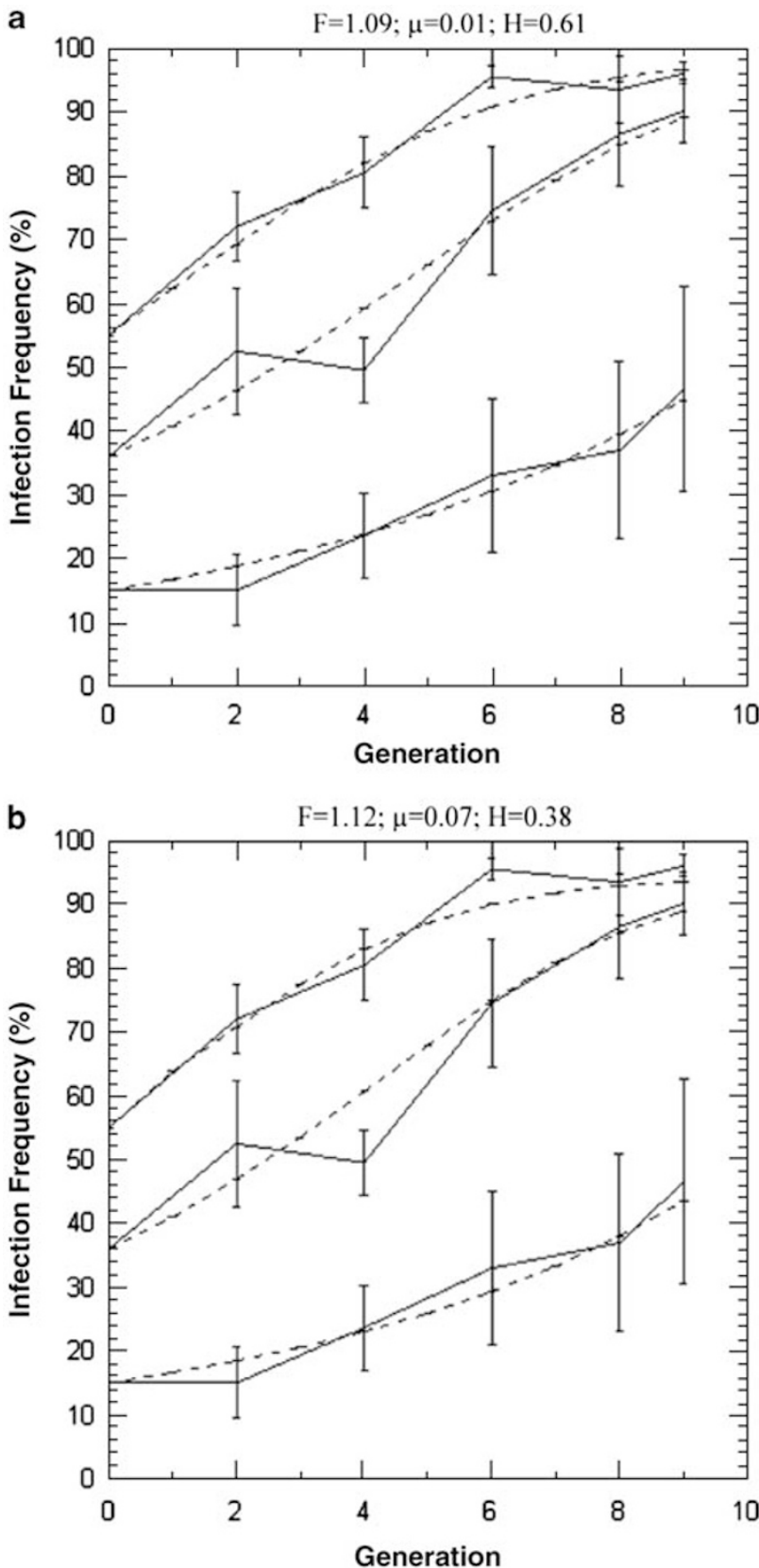

Figure 2 Spread of Cardinium using model parameter values (dotted lines) compared with observed infection frequencies in population cages (solid lines). Data represent the mean \pm standard error, $n=4$. (a) Model of best fit where the fitness benefit $F=1.09$; the proportion of offspring produced by the incompatible cross relative to the compatible cross $H=0.61$ and infidelity of maternal transmission $\mu=0.01$. (b) Model of best fit when $H$ is fixed at 0.38 (determined experimentally); fitness benefit $F=1.12$ and infidelity of maternal transmission $\mu=0.07$.

found in populations infected with a CI-inducing Wolbachia. For example, in their population cage study, Nigro and Prout (1990) showed that a Wolbachia causing CI in Drosophila simulans increased in prevalence from an initial infection frequency of $20 \%$ within a few generations. Similarly, a Wolbachia causing CI in Drosophila melanogaster increased in prevalence from $50 \%$ to near fixation within five generations (Reynolds and Hoffmann, 2002). Turelli and Hoffmann (1991) also reported
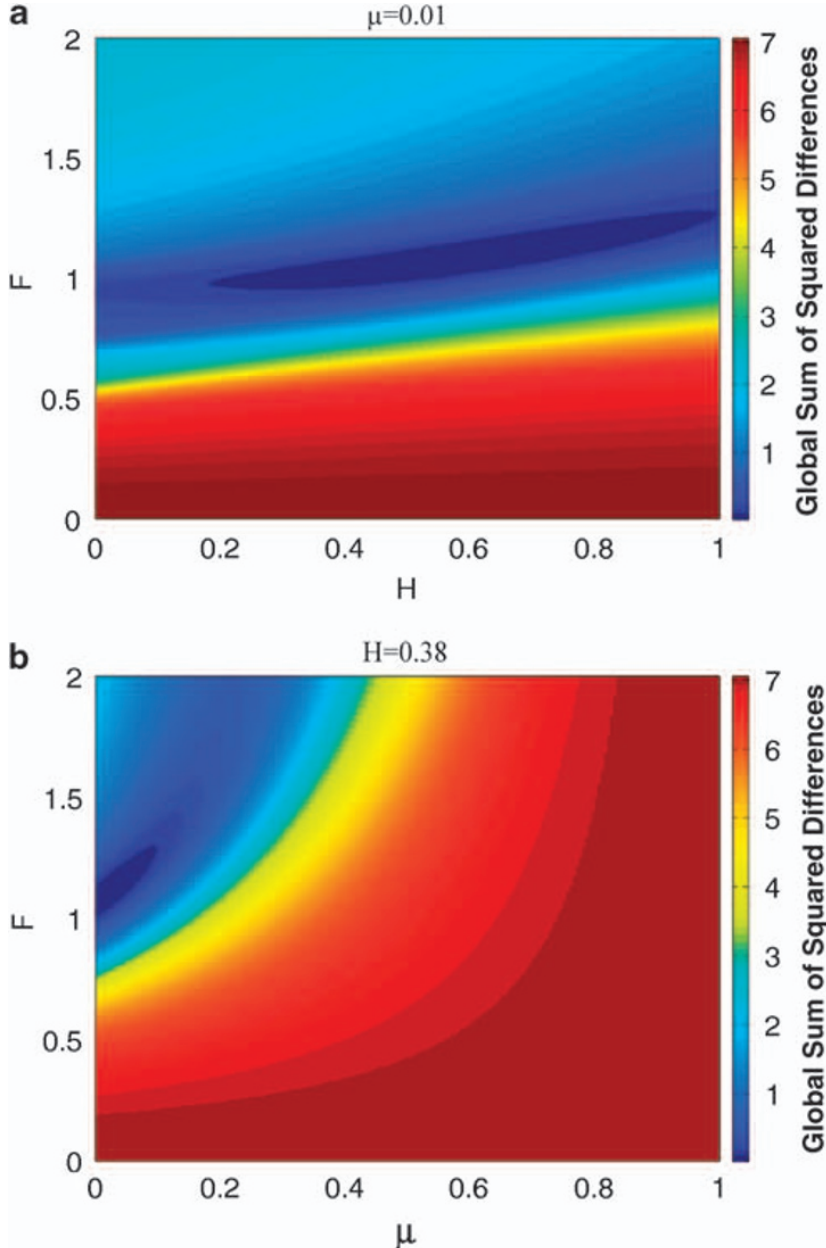

Figure 3 Sum of squared differences between observed infection frequencies and those predicted by a range of parameter values. (a) $\mu$ is fixed at 0.01 and (b) $H$ is fixed at 0.38 (as determined experimentally). Best fits (i.e., lowest sums of squares) are in blue.

the rapid geographical spread of Wolbachia in wild populations of $D$. simulans across California, where the infection was found to spread upward of $100 \mathrm{~km}$ per year. In addition, infection parameters estimated from field-collected Culex pipiens predicted the rapid spread of a CI Wolbachia to near fixation (Rasgon and Scott, 2004). Invasion thresholds were clearly evident in other studies. For example, a Wolbachia strain inducing CI in Aedes aegypti did not spread when initial infection frequencies were below 20\% (Xi et al., 2005). In addition, Johanowicz and Hoy (1999) found that a Wolbachia associated with CI in the mite Metaseiulus occidentalis was not able to spread within 12 generations when started at an introductory frequency of $10 \%$, likely because of high fitness costs of the infection in this host. Interestingly, M. occidentalis has since been found to be infected with Cardinium as well, and it is not clear which symbiont (or both) causes CI (Weeks et al., 2003).

Stochastic factors are likely responsible for some of the variation in infection cage frequencies in our study (Figure 1). For example, random sampling effects could have caused the upsurge in infection frequency in one of the cages in the medium treatment in generation 2 , as it could explain jumps or dips in infection frequencies in nature. Stochastic events may allow infections to spread 
even when the initial frequency is lower than the invasion threshold, particularly in the case of a small or subdivided population (Jansen et al., 2008). However, it is unlikely that random genetic drift was responsible for the spread of the infection in all replicate cages that were initiated below the predicted threshold.

Interestingly, the strength of CI in Cardinium-infected E. pergandiella was found to be much weaker with $H=0.38$ in this study compared with previous studies, where $H$ ranged from 0.07 to 0.13 (Hunter et al., 2003; Perlman et al., 2008). The best model fit was found to have an even weaker CI strength, at $H=0.61$ (Figure 1). Several factors have been found to affect the expression of CI, including bacterial density (Noda et al., 2001; Clark et al., 2003), temperature (Clancy and Hoffmann, 1998), male age (Turelli and Hoffmann, 1995) and host density (Sinkins et al., 1995). Although temperature and male age were similar between experiments, differences in infection density, host density or plant quality could have been important factors affecting the change in CI strength.

It is also possible that the host or symbiont has evolved toward weaker CI. Although a decrease in CI strength over time has not, to our knowledge, been reported before, theory suggests that host selection will favor variants that induce weaker $\mathrm{CI}$ if they also exhibit reduced fitness costs in infected females (Turelli, 1994; Vavre et al., 2003). A recent study showed rapid evolution in $D$. simulans infected with Wolbachia, with a shift from a 15-20\% fecundity cost to a $10 \%$ fecundity benefit within 20 years (Weeks et al., 2007). Backcrossing experiments indicate that this change in fitness effects was not due to changes in host genotype, which therefore suggests that the Wolbachia strain had evolved (Weeks et al., 2007). Although it is possible that hostparasite evolution may also have resulted in a change in CI strength or host fitness in Cardinium-infected E. pergandiella, it seems unlikely that these differences would have evolved within a period of only 5 years.

Changes in the host fitness effects of Cardinium had a large impact on fitting the model to the observed data. The best overall model fit to the observed data used a slight fitness benefit of $F=1.09$, and a fitness benefit of $F=1.12$ was found to fit optimally when $H$ was fixed at 0.38 (Figure 2). A good fit to the observed data (that is, sum of squares $<1$ ) was found using values of $F$ ranging from a slight fecundity cost $(F \sim 0.95)$ to a fecundity benefit ( $F \sim 1.30$; Figure 3$)$. In contrast, Perlman et al. (2008) reported that Cardinium infection reduced E. pergandiella host fecundity by approximately $18 \%$ within the first 4 days of reproduction $(F=0.82)$. In that study, female E. pergandiella were provided with highdensity arenas of unlimited whitefly hosts. It is likely that this observed fitness cost is not realized in circumstances where hosts are scarce. In addition, cryptic fitness benefits of the infection, such as an increased ability to find hosts or an increased fertilization rate or competitive ability, may not have been detected in the confined arenas of the laboratory study, whereas these effects may be detected in the more natural environment of the population cages. It is not uncommon for fitness effects to vary between laboratory and more natural populations. For example, a higher fitness cost of CI symbiont infection was noted in the laboratory compared with the field for Wolbachia-infected D. simulans populations
(Hoffmann et al., 1990). Recently it has been found that a CI-inducing Wolbachia in D. melanogaster protects its host from viruses (Hedges et al., 2008; Teixeira et al., 2008), and it is therefore possible that the apparent fitness benefit of Cardinium is also related to host defense.

It is interesting to note that mutually compatible CI symbionts are expected to evolve to reduced fitness costs (or increased fitness benefits), even if this also results in a decrease in CI strength (Turelli, 1994). This is because increased host fitness will result in a greater number of infected offspring (Turelli, 1994). Thus, symbiont selection does not act directly on the strength of CI, and the symbiont is expected to ultimately evolve to be benign and beneficial (Hoffmann and Turelli, 1997).

The fitness effects of a CI infection remain notoriously difficult to estimate, often due to large discrepancies between the laboratory and the field (Hoffmann and Turelli, 1988; Hoffmann et al., 1990; Turelli and Hoffmann, 1995). Monitoring infection frequencies in population cages allows for a more realistic measure of this infection parameter, relative to single individual assays (Hoffmann and Turelli, 1988; Xi et al., 2005). Our study further shows the utility that invasion threshold experiments, coupled with current invasion models, have in estimating the fitness effects of a CI infection.

The observed data best fit a model with a slight infection benefit $(F=1.09)$, near-perfect maternal transmission $(\mu=0.01)$ and a reduction in offspring of the incompatible cross of $H=0.61$ (Figure 2). On the basis of these parameter values, there is no invasion threshold, meaning the infection is able to spread at any initial infection frequency. It would be useful to test this prediction by studying E. pergandiella populations with very low $(\sim 1-2 \%)$ initial infection frequencies to determine if Cardinium is able to spread. The stable equilibrium for these infection parameter values is 98.6\%. Because the Cardinium infection frequency was able to increase from $55 \%$ to an average of $96 \%$ within nine generations, a stable equilibrium frequency of $\sim 99 \%$ seems plausible. In the laboratory, Cardinium appears to be fixed in E. pergandiella (Hunter et al., 2003), lending further support to a stable equilibrium near fixation. In addition, the predicted stable equilibrium infection frequency of $\sim 99 \%$ is only slightly higher than the estimate of $92 \%$ (95\% confidence interval of 81.4-97.9\%) infection frequency for E. pergandiella in the field (Perlman et al., 2008).

Overall, the revised model $(F=1.09, \mu=0.01$ and $H=0.61$ ) describes the observed invasion and spread of Cardinium very well (Figure 2). This is the first study examining the invasion and spread of Cardinium, and our results indicate that its infection dynamics appear similar to those of Wolbachia. The ability of Cardinium to rapidly spread within a host population shows its potential for future CI applications. These applications include releasing mass numbers of incompatible males into a pest population, thereby reducing offspring production (Laven, 1967). In addition, virulent strains of CI that induce severe fitness costs could be used to reduce the survival of disease vectors (Rasgon et al., 2003). For example, the popcorn strain of Wolbachia has been found to reduce the life span of D. melanogaster by $50 \%$ (Min and Benzer, 1997), and was recently successfully introduced into the mosquito $A$. aegypti (McMeniman et al., 2009). Furthermore, it has been suggested that CI 
symbionts could potentially be used to drive genes of interest, for example, a gene that decreases the longevity of a vector, into a population (Beard et al., 1993). Our study suggests that, in addition to Wolbachia, the symbiont Cardinium may be a good candidate for use in these potential applications.

\section{Conflict of interest}

The authors declare no conflict of interest.

\section{Acknowledgements}

We thank Josh Garcia, Tamica Montgomery, Hyomin Kim, Jen White, Amaranta Kozuch, Nick Doidge and Dan Fernandez for assistance in the lab and greenhouse; Pat Gregory, John Volpe, Rob McGregor and three anonymous reviewers for helpful suggestions and comments; and Amer Shreim, Laura Cowen, Finn Hamilton and Pavel Kratina for help with statistical analysis. This research was supported by an NSERC CGS to LH and an NSF DEB Grant (DEB-0542961) to MSH and SP. SP is a member of the Integrated Microbial Biodiversity Program of the Canadian Institute for Advanced Research.

\section{References}

Baumann P (2005). Biology of bacteriocyte-associated endosymbionts of plant sap sucking insects. Ann Rev Microbiol 59: 155-189.

Beard CB, O'Neill SL, Tesh RB, Richards FF, Aksoy S (1993). Modification of arthropod vector competence via symbiotic bacteria. Parasitol Today 9: 179-183.

Brelsfoard CL, Sechan Y, Dobson SL (2008). Interspecific hybridization yields strategy for South Pacific filariasis vector elimination. PLoS Negl Trop Dis 2: e129.

Buchner P (1965). Endosymbiosis of Animals with Plant Microorganisms. John Wiley and Sons: New York.

Caspari E, Watson GS (1959). On the evolutionary importance of cytoplasmic sterility in mosquitoes. Evolution 13: 568-570.

Clancy DJ, Hoffmann AA (1998). Environmental effects on cytoplasmic incompatibility and bacterial load in Wolbachiainfected Drosophila simulans. Entomol Exp Appl 86: 13-24.

Clark ME, Veneti Z, Bourtzis K, Karr TL (2003). Wolbachia distribution and cytoplasmic incompatibility during sperm development: the cyst as the basic cellular unit of $\mathrm{CI}$ expression. Mech Dev 120: 185-198.

Colgan DJ, McLauchlan A, Wilson GDF, Livingston SP, Edgecombe GD, Macaranas J et al. (1998). Histone H3 and U2 snRNA DNA sequences and arthropod molecular evolution. Aust J Zool 46: 419-437.

Duron O, Hurst GDD, Hornett EA, Josling JA, Engelstadter J (2008). High incidence of the maternally inherited bacterium Cardinium in spiders. Mol Ecol 17: 1427-1437.

Egas M, Vala F, Breeuwer JAJ (2002). On the evolution of cytoplasmic incompatibility in haplodiploid species. Evolution 56: 1101-1109.

Engelstadter J, Telschow A (2009). Cytoplasmic incompatibility and host population structure. Heredity 103: 196-207.

Gotoh T, Noda H, Ito S (2007). Cardinium symbionts cause cytoplasmic incompatibility in spider mites. Heredity 98: 13-20.

Hedges LM, Brownlie JC, O'Neill SL, Johnson KN (2008). Wolbachia and virus protection in insects. Science 322: 702.

Hilgenboecker K, Hammerstein P, Schlattmann P, Telschow A, Werren JH (2008). How many species are infected with Wolbachia?-a statistical analysis of current data. FEMS Microbiol Lett 281: 215-220.
Hoffmann AA, Turelli M (1988). Unidirectional incompatibility in Drosophila simulans: inheritance, geographic variation and fitness effects. Genetics 119: 434-444.

Hoffmann AA, Turelli M (1997). Cytoplasmic incompatibility in insects. In: O'Neill SL, Hoffmann AA, Werren JH (eds). Influential Passengers: Inherited Microorganisms and Invertebrate Reproduction. Oxford University Press: Oxford, pp 42-80.

Hoffmann AA, Turelli M, Harshman LG (1990). Factors affecting the distribution of cytoplasmic incompatibility in Drosophila simulans. Genetics 126: 933-948.

Hunter MS, Woolley JB (2001). Evolution and behavioral ecology of heteronomous aphelinid parasitoids. Ann Rev Entomol 46: 251-290.

Hunter MS, Perlman SJ, Kelly SE (2003). A bacterial symbiont in the Bacteroidetes induces cytoplasmic incompatibility in the parasitoid wasp Encarsia pergandiella. Proc R Soc London B 270: 2185-2190.

Jansen VAA, Turelli M, Godfray CJ (2008). Stochastic spread of Wolbachia. Proc R Soc London B 275: 2769-2776.

Johanowicz DL, Hoy MA (1999). Wolbachia infection dynamics in experimental laboratory populations of Metaseiulus occidentalis. Entomol Exp Appl 93: 259-268.

Laven H (1967). Eradication of Culex pipiens fatigans through cytoplasmic incompatibility. Nature 261: 383-384.

McMeniman CJ, Lane RV, Cass BN, Fong AWC, Sidhu M, Wang Y-F et al. (2009). Stable introduction of a life-shortening Wolbachia infection into the mosquito Aedes aegypti. Science 323: 141-144.

Mardulyn P, Cameron SA (1999). The major opsin in bees (Insecta: Hymenoptera): a promising nuclear gene for higher level phylogenetics. Mol Phylogenet Evol 12: $168-172$.

Min K-T, Benzer S (1997). Wolbachia, normally a symbiont of Drosophila, can be virulent, causing degeneration and early death. Proc Natl Acad Sci USA 94: 10792-10796.

Moran NA, McCutcheon JP, Nakabachi A (2008). Genomics and evolution of heritable bacterial symbionts. Ann Rev Genet 42: 145-190.

Nigro L, Prout T (1990). Is there selection on RFLP differences in mitochondrial DNA? Genetics 125: 551-555.

Noda H, Koizumi Y, Zhang Q, Deng K (2001). Infection density of Wolbachia and incompatibility level in two planthopper species, Laodelphax striatellus and Sogatella furcifera. Insect Biochem Mol Biol 31: 727-737.

Oliver KM, Russell JA, Moran NA, Hunter MS (2003). Facultative bacterial symbionts in aphids confer resistance to parasitic wasps. Proc Natl Acad Sci USA 100: 1803-1807.

Perlman S, Kelly SE, Hunter MS (2008). Population biology of cytoplasmic incompatibility: maintenance and spread of Cardinium symbionts in a parasitic wasp. Genetics 178: 1003-1011.

Rasgon JL (2008). Using predictive models to optimize Wolbachia based strategies for vector-borne disease control. Adv Exp Med Biol 627: 114-125.

Rasgon JL, Scott TW (2004). Wolbachia and cytoplasmic incompatibility in the California Culex pipiens mosquito species complex: parameter estimates and infection dynamics in natural populations. Genetics 165: 2029-2038.

Rasgon JL, Styer LM, Scott TW (2003). Wolbachia-induced mortality as a mechanism to modulate pathogen transmission by vector arthropods. J Med Entomol 40: 125-132.

Reynolds KT, Hoffmann AA (2002). Male age, host effects and the weak expression or non-expression of cytoplasmic incompatibility in Drosophila strains infected by maternally transmitted Wolbachia. Genet Res 80: 79-87.

Ros VID, Breeuwer JAJ (2009). The effects of, and interactions between, Cardinium and Wolbachia in the doubly infected spider mite Bryobia sarothamni. Heredity 102: 413-422.

Scarborough CL, Ferrari J, Godfray HCJ (2005). Aphid protected from pathogen by endosymbiont. Science 310: 1781. 
Sinkins SP, Braig HR, O'Neill SL (1995). Wolbachia superinfection and the expression of cytoplasmic incompatibility. Proc R Soc London B 261: 325-330.

Sinkins SP, Curtis CF, O'Neill SL (1997). The potential application of inherited symbiont systems to pest control. In: O'Neill, SL, Hoffmann AA, Werren JH (eds). Influential Passengers: Inherited Microorganisms and Invertebrate Reproduction. Oxford University Press: Oxford, pp 155-175.

Stouthamer R, Breeuwer JAJ, Hurst GDD (1999). Wolbachia pipientis: microbial manipulator of arthropod reproduction. Ann Rev Microbiol 53: 71-102.

Teixeira L, Ferreira A, Ashburner M (2008). The bacterial symbiont Wolbachia induces resistance to RNA viral infections in Drosophila melanogaster. PLoS Biol 6: 2753-2763.

Turelli M (1994). Evolution of incompatibility-inducing microbes and their hosts. Evolution 48: 1500-1513.

Turelli M, Hoffmann AA (1991). Rapid spread of an inherited incompatibility factor in California Drosophila. Nature 353: 440-442.

Turelli M, Hoffmann AA (1995). Cytoplasmic incompatibility in Drosophila simulans: dynamics and parameter estimates from natural populations. Genetics 140: 1319-1338.

Vavre F, Fouillet P, Fleury F (2003). Between- and within-host species selection on cytoplasmic incompatibility-inducing Wolbachia in haplodiploids. Evolution 57: 421-427.

Vavre F, Fleury F, Varaldi J, Fouillet P, Bouletreau M (2000). Evidence for female mortality in Wolbachia-mediated cyto- plasmic incompatibility in haplodiploid insects: epidemiologic and evolutionary consequences. Evolution 54: 191-200.

Weeks AR, Velten R, Stouthamer R (2003). Incidence of a new sex-ratio-distorting endosymbiotic bacterium among arthropods. Proc R Soc London B 270: 1857-1865.

Weeks AR, Turelli M, Harcombe WR, Reynolds KT, Hoffmann AA (2007). From parasite to mutualist: rapid evolution of Wolbachia in natural populations of Drosophila. PLoS Biol 5: e114.

Werren JH (1997). Biology of Wolbachia. Ann Rev Entomol 42: 587-609.

Werren JH, Windsor D, Guo L (1995). Distribution of Wolbachia among neotropical arthropods. Proc $R$ Soc London $B$ 262: 197-204.

White JS, Kelly SE, Perlman SJ, Hunter MS (2009). Cytoplasmic incompatibility in the parasitic wasp Encarsia inaron: disentangling the roles of Cardinium and Wolbachia symbionts. Heredity 102: 483-489.

Xi Z, Khoo CCH, Dobson SL (2005). Wolbachia establishment and invasion in an Aedes aegypti laboratory population. Science 310: 326-328.

Zabalou S, Riegler M, Theodorakopoulou M, Stauffer C, Savakis C, Bourtzis K (2004). Wolbachia-induced cytoplasmic incompatibility as a means for insect pest population control. Proc Natl Acad Sci USA 101: 15042-15045.

Zchori-Fein E, Perlman SJ (2004). Distribution of the bacterial symbiont Cardinium in arthropods. Mol Ecol 13: 2009-2016. 